\title{
Both Methanol and Water Mixture Extracts of Panax Notoginseng Flower Affect Platelet Function and Thrombus Growth by Down-Regulating PI3K/AKT and MAPKs Signaling Pathways
}

\section{Xiao Zuo}

School of Public Health (Shenzhen), Sun Yat-sen University, Guangzhou, Guangdong Province 510080 Nan Qin

Department of Clinical laboratory, The Second Affiliated Hospital of Guangzhou Medical University, Guangzhou, Guangdong Province 510260

\section{Yu-Heng Mao}

School of Public Health (Shenzhen), Sun Yat-sen University, Guangzhou, Guangdong Province 510080 Lijuan Ma

State Key Laboratory of Quality Research in Chinese Medicine, Institute of Chinese Medical Sciences, University of Macau, Taipa, Macao, 999078

\section{Qing Li}

Department of Food and Nutrition Sciences, Life Science, MMW505, The Chinese University of Hong Kong, Shatin, N.T.

\section{Zezhong Tian}

School of Public Health (Shenzhen), Sun Yat-sen University, Guangzhou, Guangdong Province 510080

\section{Mingzhu Zhao}

School of Public Health (Shenzhen), Sun Yat-sen University, Guangzhou, Guangdong Province 510080 Qiuhua Ji

School of Public Health (Shenzhen), Sun Yat-sen University, Guangzhou, Guangdong Province 510080 Yiting Chen

School of Public Health (Shenzhen), Sun Yat-sen University, Guangzhou, Guangdong Province 510080 Jian-Bo Wan

State Key Laboratory of Quality Research in Chinese Medicine, Institute of Chinese Medical Sciences, University of Macau, Taipa, Macao, 999078

Yan Yang ( $\sim$ yangyan3@mail.sysu.edu.cn)

School of Public Health (Shenzhen), Sun Yat-sen University, Guangzhou, Guangdong Province 510080

\section{Research Article}

Keywords: Chinese herbal medicine, Ginseng, platelets, saponins, thrombosis 
Posted Date: February 3rd, 2022

DOI: https://doi.org/10.21203/rs.3.rs-1301165/v1

License: (c) (i) This work is licensed under a Creative Commons Attribution 4.0 International License. Read Full License 


\section{Abstract}

Background: Platelet dysfunction plays a central role in the pathogenesis of cardiovascular diseases (CVDs). We previously found that two sapon in monomers isolated from Panax notoginseng flowers (PNF) have shown to exhibit anti-platelet activity, and here we used methanol and water extraction methods to gain two mix extracts of PNF (PNFM and PNFW) and further investigated the effects and the underlying mechanisms of PNFM and PNFW on platelet functions and thrombosis.

Methods: Healthy human PRP or gel-filtered platelets were collected for in vitro assay the PNFM and PNFW on the function and activation of platelets, while FeCl3-injured thrombosis formation in mice mesenteric arteriole was applied for in vivo assessment. Western blot analysis was applied to study the mechanic pathways.

Results: The results showed that both PNFM and PNFW significantly inhibited human platelet aggregation and activation stimulated with either ADP or thrombin with a dose-effect relationship, and inhibited platelet granule-secretion and spreading in vitro. Furthermore, PNFM and PNFW attenuated thrombus formation in mice. The effects might be associated with inhibition of platelet PI3K, Akt, P38, JNK and Erk phosphorylation.

Conclusion:These findings suggest that PNF mixture extracts could be developed as a functional ingredient to improve cardiovascular health through inhibiting platelet hyper reaction.

\section{Introduction}

Cardiovascular diseases (CVDs), including atherosclerosis and thromboembolic diseases, have become the leading causes of disease burden and death globally (G. B. D. Risk Factors Collaborators, 2017; G. B. D. Risk Factor Collaborators, 2018; 2018). Platelets, the small versatile anucleate cells, play a central role in both the progression of atherosclerotic plaques and thrombus formation (X. R. Xu et al., 2016).Excessive platelet activation, granule release, and aggregation are key events during thrombogenesis (Jagadeeswaran, Cooley, Gross, \& Mackman, 2016). Platelet activation is mediated by a variety of extracellular stimuli such as thrombinandadenosine 5'-diphosphate (ADP), which bind to Gprotein coupled receptors thus leading to subsequent activation of intracellular downstream signaling molecules (Kunapuli et al., 2003). Phosphoinositide 3-kinase (PI3K) pathway and the mitogen-activated protein kinases (MAPK) pathway are two common pathways contributing to platelet activation(Z. Li, Delaney, O'Brien, \& Du, 2010).

Due to the critical role of platelet in thrombus formation, antiplatelet therapy has become a useful strategy to prevent acute thromboembolic artery occlusions in cardiovascular diseases(Schror, 1995; Sharma \& Berger, 2011). However, most of the antiplatelet agents may lead to severe adverse effects, especially under a high dosage or a long course of treatment. The increasing bleeding risk limited the clinical applications of many antiplatelet medicines (Michelson, 2010). Therefore, the development of novel natural antiplatelet agents without bleeding risk have been attracted the increasing attention. 
Panax notoginseng, a well-known and valuable Chinese medicine, has been widely used for the treatment of CVD more than 400 years(L. Wang et al., 2013). Among the different parts of the plant, P. notoginseng flowers (PNF) contain the highest abundance of saponins. A numerous studies indicated that PNF exert the beneficial effects on hypertension, insomnia, and stomatitis. Our previous study reported that two monomers (G-Rb2 and G-Rd2) from PNF significantly inhibited human platelet aggregation and activation induced by adenosine diphosphate (ADP) in vitro. However, the effects of PNF mixture extracts on platelet function were unclear yet.

Methanol and water are the two most common solvents to extract the bioactive compounds of herbal medicine. Our previous studies have demonstrated that ginsenoside profiles of PNF (Ma, Liu, Zhong, \& Wan, 2017) or $P$. notoginseng leaves (Sun, Ma, Wan, \& Tong, 2021) extracted by methanol and water were remarkably different, and the difference between the two extracts may due to the ginsenoside transformations happennedduring the water extraction process. Specifically, we found that ginsenoside Rb1 (G-Rb1, 1), G-Rc (2), G-Rb2 (3), G-Rb3 (4) and G-Rd (5), the major saponins of PNF, can be largely converted into gypenoside XVII (GY-XVII, 6), notoginsenoside Fe (NG-Fe, 7), G-Rd2 (8) and NG-Fd (9) and G-F2 (10), respectively, through selectively cleaving the $\beta$ - $(1 \rightarrow 2)$ - glucosidic linkage at the C-3 site of ginsenosides without hydrolyzing other glycosidic linkages (Ma, Ma, Cao, \& Wan, 2022). It was proposed that PNF extracted by methanol (PNFM) and water (PNFW) may exert different healthy effects due to their different components (Ma et al., 2017). The aim of the present study, therefore, was to investigate the effects of two mixture extracts PNFM and PNFW on human platelet functions and the underlying mechanisms.

\section{Materials And Methods}

\subsection{Chemicals and antibodies}

Chemical reference standards, including G-Rb1 (1) and NG-Fe (8), were supplied by National Institute for the Control of Pharmaceutical and Biological Products (Beijing, PR China). G-Rc (2), G-Rb2 (4), and G-Rb3 (5) were purchased from Must Bio-technology (Chengdu, PR China). NG-Fc (3), NG-Fd (10), G-Rd2 (9), and GY-XVII (7) were supplied by Baoji Herbest Bio-Tech (Baoji, PR China). The purity of each reference standard was over $98 \%$ measured by HPLC-UV. LC-grade acetonitrile and methanol were purchased from Merk (Darmstadt, Germany). ADP and thrombin were purchased from Sigma-Aldrich (St. Louis, MO, USA). Antibodies forFITC-conjugated anti-human CD62P, PAC-1 and IgG1 isotype control were purchased from BD Biosciences (San Jose, CA, USA). Alexa FluorTM 488-Conjugated anti-human fibrinogen antibody was purchased from Thermo Fisher Scientific (Waltham, MA, USA). Antibodies for $\beta$-actin, phospho-Akt $\left(\mathrm{Ser}^{473}\right)$, phosphor-PI3k $\left(\mathrm{Tyr}^{458}\right)$, phospho-p38 $\left(\mathrm{Thr}^{180} / \mathrm{Tyr}^{182}\right)$, phospho-ERK1/2 $\left(\mathrm{Thr}^{202} / \mathrm{Tyr}^{204}\right)$, and phospho-JNK (Thr ${ }^{183} /$ Tyr $^{185}$ ) were purchased from Cell Signaling Technology (Beverly, MA, USA). The ultra-pure water $\left(18.2 \mathrm{M} \Omega . \mathrm{cm}\right.$ at $25^{\circ} \mathrm{C}$ ) was purified by a Milli-Q purification system (Millipore, Bedford, MA, USA).

\subsection{Preparation of PNF extracts and Chemical analysis}


PNF was purchased from a drug store in Kunming, Yunnan Province, China. Their botanical origin of materials was authenticated by Professor Ni Ma from WenshanSanqi Institute of Science and Technology, Yunnan, China. The preparation of PNFM and PNFW was performed as described previously with slight modification (Ma et al., 2017).Approximate $36 \mathrm{~g}$ of PNF fine powder was ultrasonically (135 W) extracted by $360 \mathrm{~mL}$ of methanol or water for $2 \mathrm{~h}$ in an ultrasonic bath ( $40 \mathrm{kHz}$, Bransonic, USA). After filtration, the extracts were concentrated under the reduced pressure in a rotary vacuum evaporator at $50^{\circ} \mathrm{C}$ followed by lyophilization. The freeze-dried extracts were reconstituted in distilled water for the following in vivo study.

The chromatographic analysis was performed on anAgilent1290InfinityllUPLC system (Agilent Technologies, Santa Clara, CA, USA), equipped with an Agilent 1290 DAD. The separation was achieved on a Kinetex C18 coumn (100 mm $\times 4.6 \mathrm{~mm}, 2.6 \mu \mathrm{m}$, Phenomenex). The mobile phases were consisted of water $(A)$ and acetonitrile $(B)$ at a flow rate of $0.6 \mathrm{~mL} / \mathrm{min}$. The linear gradient program was adopted as follows: 0-5 min, 34\% B; 5-6 min, 36-38.5\% B; 6-12 min, 38.5-45\% B; $12-12.5 \mathrm{~min}, 34 \% \mathrm{~B}$. The detection wavelength was set at $203 \mathrm{~nm}$ and the column temperature was kept at $40^{\circ} \mathrm{C}$. Each sample was filtered through a $0.22 \mu \mathrm{m}$ membrane before the injection. The injection volume was $2 \mu \mathrm{L}$.

\subsection{Human blood preparation}

Healthy male and female volunteers aged between 25 to 40 years who had not taken any antiplatelet drugs within the previous 2 weeks wererecruited for this study. Written informed consents were obtained from all subjects. This study was approved by the Ethics Committee of School of Public Health, Sun Yatsen University [(2019) No. 134]. The anticoagulated whole human blood was collected to prepare plateletrich plasma (PRP) and gel-filtered platelets as our previously described (Song et al., 2014; Ya et al., 2019; Yao et al., 2017).

\subsection{Lactate dehydrogenase activity determination}

To evaluate the platelet cytotoxicity, the leakage of lactate dehydrogenase (LDH) was determined by LDH assay kit (Beyotime Institute of Biotechnology, Jiangsu, China) according to the manufacturer's instruction. Gel-filtered platelets $\left(1 \times 10^{8}\right.$ platelets $\left./ \mathrm{mL}\right)$ were pre-incubated with PNFM or PNFW $(0-2000$ $\mu \mathrm{g} / \mathrm{mL}$ ) for $20 \mathrm{~min}$, and then centrifuged at $3000 \mathrm{rpm}$ for $5 \mathrm{~min}$ at room temperature. LDH released was expressed as percentage (\%) compared with the positive control ( $0.1 \%$ triton) lysed by LDH release reagent treatment.

\subsection{Assay of platelet aggregation}

Human PRP or gel-filtered platelets were incubated with PNFM or PNFW at different concentrations (100, 300 , and $500 \mu \mathrm{g} / \mathrm{mL}$ ) or the control buffer for $20 \mathrm{~min}$ at $37^{\circ} \mathrm{C}$ according to previous study (Zuo et al., 2021). Platelet aggregation was induced at $37^{\circ} \mathrm{C}$ with a sample stir speed of $1000 \mathrm{rpm}$ in an aggregometry (Chrono-Log, Havertown, PA) using ADP (5 and $10 \mu \mathrm{M})$ or thrombin $(0.5 \mathrm{U})$ as the agonists. Aggregation was recorded for 6 min as our previously described methods (Song et al., 2014). 


\subsection{Flow cytometry analysis of CD62P expression, PAC-1 binding and fibrinogen binding to activated platelets}

PRP and gel-filtered platelets were incubated with different concentrations of PNFM, PNFW or the control

buffer at $37^{\circ} \mathrm{C}$ for $20 \mathrm{~min}$. Aliquots of sample $\left(5 \times 10^{5}\right.$ platelets) were incubated with FITC-conjugated antihuman CD62P and PAC-1 antibodies, Alexa FluorTM 488-Conjugated anti-human fibrinogen binding antibody or FITC-conjugated anti-human IgG1 antibody (isotype control), respectively, at room temperature for $30 \mathrm{~min}$. PRP were activated with $200 \mu \mathrm{M}$ ADP for 5 min while gel-filtered platelets with $0.5 \mathrm{U} / \mathrm{mL}$ thrombin in the presence of $1 \mathrm{mM} \mathrm{Ca}^{2+}$ for $5 \mathrm{~min}$. The platelets were fixed with $1 \%$ paraformaldehyde before flow cytometry analysis.

\subsection{Platelet ATP release assay}

The secretion of ATP was determined in a Chrono-log lumiaggregometer according to the manufacturer's instructions. Briefly, PRP at $2.5 \times 10^{8}$ platelets $/ \mathrm{mL}$ were incubated with different concentrations of PNFM, PNFW and the control buffer at $37^{\circ} \mathrm{C}$ for $20 \mathrm{~min}$ as described above. Luciferin-luciferase reagent was added directly to platelet suspensions, which were continually stirred at $1,000 \mathrm{rpm}$ at $37^{\circ} \mathrm{C} .5 \mu \mathrm{M}$ or 10 $\mu \mathrm{M}$ ADP was added to activate platelets and real-time ATP secretion was monitored.

\subsection{Assay of soluble $\beta$-thromboglobulin ( $\beta$-TG)}

To detect platelet $\beta$-TG in vitro, PRP $\left(2.5 \times 10^{8}\right.$ platelets $\left./ \mathrm{mL}\right)$ was pre-incubated with different concentrations of PNFM, PNFW or control buffer for $20 \mathrm{~min}$ at $37^{\circ} \mathrm{C}$ as described, and then stimulated with $5 \mu \mathrm{M}$ ADP, followed by centrifugation at $10000 \times \mathrm{g}$ for $5 \mathrm{~min}$ at $4^{\circ} \mathrm{C}$. The cell-free supernatant was collected by a new clean tube and stored at $-80^{\circ} \mathrm{C}$ until use. $\beta$-TG levels in supernatant were determined using a $\beta$-TG ELISA kit (BlueGene Biotech, Shanghai, China) according to the manufacturer's instruction.

\subsection{Assay of platelet $\mathrm{Ca}^{2+}$ mobilization}

The intracellular calcium ion concentration was measured using Fluo-3AM as a calcium ion fluorescence probe. Briefly, PRP $\left(2.5 \times 10^{8}\right.$ platelets $\left./ \mathrm{mL}\right)$ was incubated with $10 \mu \mathrm{M}$ Fluo-3AM for $30 \mathrm{~min}$ at $37^{\circ} \mathrm{C}$. The Fluo-3-loaded platelets were pre-incubated with different concentrations of PNFM or PNFW for 20 min at $37^{\circ} \mathrm{C}$ in the presence of $1 \mathrm{mM} \mathrm{CaCl}_{2}$, and then stimulated with $200 \mu \mathrm{M} \mathrm{ADP}$ or $0.5 \mathrm{U}$ thrombin. Fura-3 fluorescence in the cytosol was measured by a spectrofluorometer as the following formula: $\left[\mathrm{Ca}^{2+}\right] \mathrm{i}(\mathrm{nM})$ $=224 \times\left(F-F_{\min }\right) /\left(F_{\text {max }}-F\right)$, where 224 is the dissociation constant of the Fura-3-Ca ${ }^{2+}$ complex, and $F_{\min }$ and $\mathrm{F}_{\max }$ are the fluorescence intensities at very low and very high $\mathrm{Ca}^{2+}$ concentrations, respectively.

\subsection{Platelet spreading on immobilized Fibrinogen}

Chamber slides with microtiter wells were coated with $100 \mu \mathrm{g} / \mathrm{mL}$ fibrinogen overnight at $4^{\circ} \mathrm{C}$. PRP were incubated with different concentrations of PNFM, PNFW and the control buffer for 20 min at $37^{\circ} \mathrm{C}$ as described above. The platelets were allowed to adhere and spread on fibrinogen-coated wells at $37^{\circ} \mathrm{C}$ for 
$1 \mathrm{~h}$. After washing, the cells were fixed, permeabilized, and stained with Alexa Fluor 488-conjugated phalloidin before observing with an inverted fluorescence microscope. The spreading areas of single platelet were measured using ImageJ software. Ten randomly selected fields from at least three parallel tests were used for statistical analysis.

\subsection{Western blot analysis}

After incubation with PNFM, PNFW and the control buffer for 20 min,platelets were activated with ADP (5 $\mu \mathrm{M})$ or Thrombin $(0.5 \mathrm{U})$ in the presence of $1 \mathrm{mM} \mathrm{Ca}^{2+}$ for $5 \mathrm{~min}$. Platelet were harvested and lysed with RIPA buffer supplemented with protease and phosphatase inhibitors for $30 \mathrm{~min}$ on ice. After centrifugation at $12000 \mathrm{~g}$ for $15 \mathrm{~min}$. the supernatants were collected as platelet total protein for western blot analysis. The protein concentrations were determined using a commercial BCA kit (Thermo Fisher Scientific, Rockford, IL). Equal amounts of protein $(20-50 \mu \mathrm{g})$ were fractionized on 10\% SDS-PAGE gels and transferred onto a polyvinylidene difluoride (PVDF) membrane. The membranes were blocked with $5 \%$ nonfat milk in TBST and then incubated with each primary antibodies including $\beta$-actin, GAPDH, phospho-PI3K, phospho-Akt, phospho-Erk1/2, phospho-JNK, phospho-p38 and corresponding secondary antibodies. The target proteins were detected with an enhanced chemiluminescence (ECL) reagent (Thermo Scientific, Waltham, MA, USA) in an automatic chemiluminescence image analysis system.

\subsection{Assay of $\mathrm{FeCl}_{3}$-injured thrombosis formation in mice mesenteric arteriole}

Intravital microscopy of $\mathrm{FeCl}_{3}$-induced thrombus formation in 3 to 4 -week male $\mathrm{C} 57 \mathrm{BL} / 6 \mathrm{~J}$ mice mesenteric arteriole was performed as described previously (Zuo et al., 2021). The animal experiments were approved by the Animal Care and Use Committee of Sun237 Yat-sen University (No. SYXK [Yue] 2017-0080). Briefly, after injection of PNFM, PNFW (30 $\mathrm{g} / \mathrm{g} \mathrm{BW),} \mathrm{or} \mathrm{control} \mathrm{buffer} \mathrm{via} \mathrm{the} \mathrm{tail} \mathrm{vein} \mathrm{followed}$ by inject calcein-labeled $(4 \mu \mathrm{g} / \mathrm{mL})$ mice platelets. The dose of PNFM and PNFW in animal experiments was calculated according to the dose of in vitroexperiments, approximately $500 \mu \mathrm{g} / \mathrm{mL}$ as the final concentration in the murine blood. The injury was induced by topical application of $\mathrm{FeCl}_{3}$. Images of thrombus formation and dissolution were visualized by a fluorescence microscope (Leica Microsystems, Wetzlar, Hesse, Germany). Based on the time to complete the vessel occlusion, the images from each group are compared with thosefrom the other groups.

\subsection{Assay of bleeding time in mice}

Male C57BL/6J mice (6-8 weeks old) were injected with PNFM, PNFW (30 $\mu \mathrm{g} / \mathrm{g} \mathrm{BW}$ ) or the control buffer via the tail vein 20 min before the bleeding time assay. Mice were then anesthetized with sodium pentobarbital and maintained at $37^{\circ} \mathrm{C}$ on a heating pad during the experiment. The $5 \mathrm{~mm}$ tip of tail was cut off and placed into $37^{\circ} \mathrm{C}$ saline solutions immediately. The bleeding time was calculated from the moment blood began emerging to the moment bleeding ceased.

\subsection{Statistical analysis}


GraphPad Prism version 5.01 software (GraphPad Inc., San Diego, CA, USA) was used for statistical analyses. Data are expressed as the means \pm standard deviation (SD) of at least three independent experiments. The statistical significance among different groups was determined using one-way ANOVA. Differences were considered significant at $P<0.05$.

\section{Results}

\subsection{Chromatographic analysis of major ingredients}

The contents of abundant saponins, G-Rb1, G-Fc, G-Rc, G-Rb2, G-Rb3, GY-XVII, NG-Fe, G-Rd2 and NG-Fd in water extracts and methanol extractsof PNF were determined by an optimized UPLC-UV chromatographic condition. The saponins in different PNF extracts were well separated (Fig. 1).As shown in Fig. 1, the UPLC profiles of PNFM and PNFW were remarkably different. The G-Rb1 (1), G-Fc (2), G-Rc (3), G-Rb2 (4), and GRb3 (5) were found as the major saponins in PNFM. Compared to PNFM, the contents of G-Rb1(1), G-Rc (3), G-Rb2 (4), and G-Rb3 (5) were decreased and the contentsof GY-XVII(6), NG-Fe(7), G-Rd2(8) and NG$\mathrm{Fd}(9)$ were greatly increased after water immersion. These findings suggested that ginsenoside transformations happen upon the extraction with water.

\subsection{PNFM and PNFW inhibit human platelet aggregation in vitro}

Both PNFM and PNFW significantly inhibited ADP- and thrombin-induced human platelet aggregation in a dose-dependent manner (Fig. 2A-C). Additionally, PNFW at $300 \mu \mathrm{g} / \mathrm{mL}$ exhibited a more potent effect on the suppression platelet aggregation induced by ADP at both $5 \mu \mathrm{M}$ and $10 \mu \mathrm{M}$, compared with PNFM at the same dose (Fig.2A-B). The dosages of PNFM and PNFW used in this study had no cytotoxicity on human platelets as demonstrated by the LDH leakage assay (Supplementary Figure 1).

\subsection{PNFM and PNFW attenuate human platelet surface CD62P expression, PAC-1 binding, and platelet binding to fibrinogen in vitro}

CD62P expressed on platelet surface is a marker of platelet activation. Upon agonist stimulation, the transduction of intraplatelet signals leads PAC-1 to switch from a low-affinity to high-affinity state for fibrinogen, which initiates and amplifies platelet aggregation and thrombus consolidation (Ya et al., 2018). As shown in Fig. 3, both PNFM and PNFW dose-dependently inhibited ADP- and thrombin-induced CD62P expression, PAC-1 activation, and fibrinogen binding. We found the inhibitory effects of PNFW on human platelet CD62P expression were stronger comparing with PNFM at low concentration (100 $\mu \mathrm{g} / \mathrm{mL}$ ). On the contrary, the PNFM showed more potent inhibition on PAC-1 activation at all concentrations compared with PNFW. Additionally, the inhibitory effects of PNFM on thrombin- induced PAC-1 activation and fibrinogen binding were significantly stronger compared with PNFW at relatively higher concentrations (300 or $500 \mu \mathrm{g} / \mathrm{mL}$ ).

\subsection{PNFM and PNFW inhibit human platelet ATP release, $\beta$-TG expression, and $\mathrm{Ca}^{2+}$ mobilization in vitro}


Granule secretion is an important marker of platelet activation prior to aggregation and thrombus formation. As shown in Fig. 4A-C, both PNFM and PNFW decreased intraplatelet ATP release induced by ADP $(5 \mu \mathrm{M}$ and $10 \mu \mathrm{M})$ and thrombin $(0.1 \mathrm{U})$ in a dose-dependent manner. Both PNFM and PNFW also dose-dependently reduced the intraplatelet $\mathrm{Ca}^{2+}$ mobilization induce by ADP and thrombin (Fig. 4D-E). Additionally, the release of intraplatelet $\beta$-TG were attenuated by PNFM and PNFW at the dose of 500 $\mu \mathrm{g} / \mathrm{mL}$ (Fig. 4 F).

\subsection{PNFM and PNFW decrease human platelet spreading on immobilized fibrinogen in vitro}

After fibrinogen binding to platelet PAC-1, it transduces outside-in signals into the cell and triggers platelet spreading. We next investigated whether PNFM and PNFW influence platelet spreading on immobilized fibrinogen. As shown in Fig. 4G, PNFM and PNFW substantially decreased the surface area of the spreading platelets on immobilized fibrinogen. Additionally, the inhibitory effects of PNFM on platelet spreading were more potent than those of PNFW at relatively lower dose $(100 \mu \mathrm{g} / \mathrm{mL})$ rather than other two higher doses.

\subsection{Effects of PNFM and PNFW on phosphorylation of PI3k/Akt and MAPKs in platelets}

PI3K/Akt and MAPKs (ERK, JNK, and p38 MAPK) pathways play a crucial role in platelet activation. Our results demonstrated that both PNFM and PNFW markedly down-regulated the phosphorylation of PI3k, Akt, JNK, ERK, and p38 MAPK in ADP- and thrombin-treated human platelets in a dose-dependent manner (Fig. 5). These results suggested that the ameliorative effects of PNFM and PNFW on platelet hyperactivity were possibly mediated by concomitant inhibition of PI3k/Akt and MAPK signaling pathways.

\subsection{PNFM and PNFW inhibit $\mathrm{FeCl}_{3}$-induced mesenteric thrombus formation in mice}

The results of in vivo thrombus model experiment showed that $500 \mu \mathrm{g} / \mathrm{mL}$ of PNFM and PNFW effectively suppressed $\mathrm{FeCl}_{3}$-induced mesenteric thrombus formation as demonstrated by the prolonged vessel occlusion time (Fig. 6A-6B).We also found that PNFW exerted more potent inhibitory effects on thrombus formation than PNFM treatment. Additionally $\llbracket$ we found that compared with the control, the treatment of either PNFM or PNFW at $500 \mu \mathrm{g} / \mathrm{mL}$ had no significant influence on the tail bleeding time, which is an indicator of normal hemostasis and coagulation functions (Fig.6 C).

\section{Discussion}

Resting platelets circulate in the form of small discs without interacting with each other or the vascular endothelium (Blache, 1992). Upon vascular injury, platelets adhere to the subendothelial matrix becoming activated and aggregated (Z. Li et al., 2010). Platelets could be activated by various extracellular stimuli, including ADP and thrombin.ADP activates platelets mainly via the $\mathrm{P}_{2} \mathrm{Y}_{1}$ and $\mathrm{P} 2 \mathrm{Y}_{12}$ receptors through positive feedback loops that amplify platelet activation (Hollopeter et al., 2001). Thrombin is regarded as the most powerful physiological platelet agonist. Thrombin could activate human platelets via a dual 
system of G-protein coupled protease-activated receptors (PAR), namely PAR1 and PAR4, which promote platelet shape change, aggregation, mobilization of P-selectin to platelet surface, integrin allb $\beta 3$ activation and granule secretion (Angiolillo, Capodanno, \& Goto, 2010). The present study showed that PNFM and PNFW effectively inhibited human platelet aggregation induced by both ADP and thrombin. Since ADP and thrombin have different receptors, we speculated that PNFM and PNFW have more comprehensive effects onplatelets via various pathways, instead of working as a single specific antagonist to inhibit the binding to platelet membrane receptors.

Although the antithrombotic benefits of P.notoginseng roots have been well documented before (Fu et al., 2021), few studies have evaluated the medicinal use of PNF for the prevention and treatment of cardiovascular diseases. Our previous studies showed that two saponin monomers (Rb2 and Rd2) in PNF significantly attenuated platelet function(Zuo et al., 2021). In the present study, we demonstrated that both the methanol (PNFM) or water aqueous (PNFW) mixture extracts from PNF can also effectively inhibited human ADP- and thrombin-induced platelet activation, granule secretion, aggregation and platelet spreading on fibrinogen in vitro. Compared with the saponin monomers that are suitable for pharmaceuticalapplication, PNF mixture extracts are more available and convenient to be utilized as a nutritional ingredient in functional food.

The pharmacological activities may differ among different ginsenosides. Our previous study has identified different ginsenoside profiles between PNFM and PNFW. The chromatographic analysis revealed that the compositional ratio of original types saponins in PNFM were obviously higher than PNFW, while the transformed types saponins were higher in PNFW than PNFM. Specifically, during the water extraction process, the $\beta$ - $(1 \rightarrow 2)$ - glucosidic linkage at the $C-3$ site of ginsenosidesis selectively cleaved while otherglycosidic linkage keeps intact. The major saponins in PNF methanol mixture extract (Fig. 1A), ginsenoside Rb1 (G-Rb1), G-Rc, G-Rb2, G-Rb3, and G-Rd were partially converted into other saponins, including gypenoside XVII (GY-XVII), notoginsenoside Fe (NG-Fe), G-Rd2 and NG-Fd and G-F2 (Fig. 1B) (Ma et al., 2017). In this study, although both PNFM and PNFW exerted significant effects on human platelet functions with similar dose-dependent trends, minor differences were found among different doses. The inhibitory effects of PNFW on human platelet aggregation, CD62p expression, platelet spreading and ATP release and thrombus formation were more potent than PNFM, while the effects of PNFM on allb 33 activation, fibrinogen binding, and $\mathrm{Ca}^{2+}$ mobilization were stronger than PNFW. The difference in the antithrombotic effects of PNFM and PNFW could be partially attributed to the different saponin profiles.Another studyalso reported that the water and methanol extracts of a traditional medicine (Ocimumamericanum) displayed different healthy effect (Zengin et al., 2019). But the specific mechanism remained to be further studied.

Notoginsenoside Fc (NG-Fc), a main composition of both PNFM and PNFW, has been shown to inhibit platelet aggregation induced by ADP, thrombin, and collagen at submaximal concentrations(Liu et al., 2018). Except NG-Fc, among the 10 saponins isolated from PNF extracts in our previous studies have indicated that, only G-Rb2, and G-Rd2 could inhibit ADP-induced platelet aggregation at the concentration of $100 \mu \mathrm{g} / \mathrm{mL}$ (Zuo et al., 2021).Additionally, in the present study, our results showed that PNFM and 
PNFW may have stronger antiplatelet effect than saponin monomers, as they were still effective with higher doses of agonists (10 $\mu \mathrm{M}$ ADP and $0.5 \mathrm{U}$ thrombin). Notably, according to the quantitative determination of the saponins in the PNFM and PNFW (Figure 1), the effective dosage of the NG-Fc, G$\mathrm{Rb} 2$, and G-Rd2 in our previous study (Zuo et al., 2021) are much higher than those saponin monomers in PNFM and PNFW. This may due to the synergistic effects of various saponin monomers in two PNF extracts, which is also consistent to a reported study that ginsenosideRp3 synergistically inhibits platelet aggregation with other ginsenosides (Irfan et al., 2018). Additionally, some other saponins with lower concentrations in PNFM and PNFW should be taken into consideration in future studies. It indicated that PNF mixture extracts at a lower dosage could confer the similar therapy compared to the saponin monomers.

Bioavailabilityand pharmacokinetics are two major issues influencing the health effects of natural compounds used as dietary supplements.A previous study indicated that NG-Fc have a slow elimination from plasma with a long $\mathrm{t}_{1 / 2}$ (approximately 22-30 $\mathrm{h}$ ) and its oral bioavailability was $0.10-0.14 \%$ (He et al., 2015). Moreover, it was reported that the oral bioavailability of Rb1 was $4.35 \%$ after administering PNS to rats and the maximal concentration of plasma G-Rb1 could be reached about $50 \mu \mathrm{M}$ after $5 \mathrm{~min}$ of intravenous dosing $5 \mu \mathrm{mol} / \mathrm{kg}$ body weight of G-Rb1 in rats (Q. F. Xu, Fang, \& Chen, 2003). Additionally, it is estimated that the serum concentration of PNS after administrating Xueshuantong® Injection (a clinical medicine in which PNS is the main component) is compatible to the concentration of $100 \mu \mathrm{g} / \mathrm{mL}$ used in thisstudy(R. L. Li et al., 2020). Although the systemic bioavailability of PNFM and PNFW are not clearly, health effects were observed in animal studies. Orally administration of $120 \mathrm{mg} / \mathrm{kg} / \mathrm{day}$ PNF extractions for 9 weeks significantly improved the ventricular hypertrophy state in mice(Y. Wang et al., 2012). In a myocardial infarction rats model, oral $1000 \mathrm{mg} / \mathrm{kg} P$. notoginseng flower decoction could relieve symptoms of MI (Zhou, Li, Chen, \& Xie, 2019). Although whether orally administration of PNFM and PNFW can modify platelet function and attenuate thrombus formation is unclear yet, we demonstrated platelet inhibitory effects of PNFM and PNFW in vitroin the present study, and the bioavailability and pharmacokinetics of PNFM and PNFW and their potential health benefits in vivo will be clarified in the further studies based on the present results.

$\mathrm{PI} 3 \mathrm{~K} / \mathrm{Akt}$ and the MAPK pathways play an important role in human platelet activation and are reported in many studies (Ma et al., 2017). The inhibition of PI3K/Akt could prevent integrin allbß3-mediated platelet adhesion and thrombus formation (Morello, Perino, \& Hirsch, 2009). MAPKs, including JNK1, ERK2, and p38, have been identified to be involved in platelet activation induced by various agonists (Bugaud, Nadal-Wollbold, Levy-Toledano, Rosa, \& Bryckaert, 1999). In the present study, we found that PNFM and PNFW significantly suppressed the activation of PI3K, Akt, and MAPKs induced by both ADP and thrombin in a dose-dependent manner. Therefore, we concluded that the inhibitory effects of PNFM and PNFW on platelet hyperactivity may partly result from concomitant inhibition of PI3K/Akt and MAPK signaling. Our results were consistent with previous study of PNF saponin monomers. G-Rb2 and G-Rd2 work on platelets by modulating P2Y12-mediated signaling in a way of up-regulating cAMP/PKA signaling and down-regulating PI3K/Akt/Erk1/2 signaling pathways(Zuo et al., 2021). 


\section{Conclusion}

In conclusion, both PNF water and methanal mixture extracts inhibited ADP- and thrombin- induced platelet aggregation, activation, granule secretion and spreading without causing bleeding risk. Their inhibitory effects on platelet function might be associated with suppressing phosphorylation of $\mathrm{PI} 3 \mathrm{~K} / \mathrm{Akt}$ and MAPKs. Compared with saponin monomers, the PNF mixture extracts are more accessible and easily produced. As natural plant extracts, they would be more acceptable by patients compared with traditional medicines. Therefore, our findings suggest that the PNF mixture extractsare valuable ingredients for application in pharmacal industry in the future and could be potentiallyapplied in preventing thrombotic and cardiovascular diseases in the future.

\section{Abbreviations}

PNF: Panax notoginseng flowers; PNFM: PNF extracted by methanol; PNFW: PNF extracted by water; HPLC: high performance liquid chromatography;

\section{Declarations}

\section{- Ethical Approval and Consent to participate}

In this study, all experiments were conducted in compliance with Chinese laws or guidelines and approved by the Ethics Committee of Sun Yat-sen University. The in vitro experiments involving healthy donors were performed in accordance with the Declaration of Helsinki guidelines, and approved by the Ethics Committee of Sun Yat-sen University [(2019) No. 134]. Singed informed consents were obtained from human participants of this study. The in vivo experiments were approved by the Animal Care and Use Committee of Sun 237 Yat-sen University (No. SYXK [Yue] 2017-0080).

\section{- Consent for publication}

Not applicable.

\section{- Availability of supporting data}

Not applicable.

\section{- Competing interests}

The authors declare that they have no known competing financial interests or personal relationships that could have appeared to influence the work reported in this paper. 


\section{- Funding}

This work was supported by the grants from National Natural Science Foundation of China (No82030098 and No. 81872617), the Sun Yat-sen University Student Innovation Training Program, the Research Committee of the University of Macau (MYRG2017-00035-ICMS), the Science and Technology Development Fund, Macau SAR (File no. 034/2019/A1), and the China Postdoctoral Science Foundation (Program No. 2020T130145ZX and Program No. 2020M673025).

\section{- Authors' contributions}

Xiao Zuo: Project administration, Methodology, Writing. Nan Qin: Methodology, Investigation. Yu-Heng Mao: Funding acquisition, Methodology, Writing. Lijuan Ma: Investigation, Visualization, Writing. Qing Li: Methodology. Zezhong Tian: Writing - review. Qiuhua Ji: Investigation. Yiting Chen: Investigation. Mingzhu Zhao: Investigation. Jian-Bo Wan: Funding acquisition, Supervision, Resources. Yan Yang: Supervision, Resources, Writing - review \& editing.

\section{- Acknowledgements}

We thank all the participants in this study.

\section{References}

1. Angiolillo, D. J., Capodanno, D., \& Goto, S. (2010). Platelet thrombin receptor antagonism and atherothrombosis. Eur Heart J, 31(1), 17-28. doi:10.1093/eurheartj/ehp504

2. Blache, D. (1992). Structure and function of blood platelets. Arch Int Physiol Biochim Biophys, 100(4), A17-24. doi:10.3109/13813459209000709

3. Bugaud, F., Nadal-Wollbold, F., Levy-Toledano, S., Rosa, J. P., \& Bryckaert, M. (1999). Regulation of cjun-NH2 terminal kinase and extracellular-signal regulated kinase in human platelets. Blood, 94(11), 3800-3805.

4. Collaborators, G. B. D. R. F. (2017). Global, regional, and national comparative risk assessment of 84 behavioural, environmental and occupational, and metabolic risks or clusters of risks, 1990-2016: a systematic analysis for the Global Burden of Disease Study 2016. Lancet, 390(10100), 1345-1422. doi:10.1016/S0140-6736(17)32366-8

5. Collaborators, G. B. D. R. F. (2018). Global, regional, and national comparative risk assessment of 84 behavioural, environmental and occupational, and metabolic risks or clusters of risks for 195 countries and territories, 1990-2017: a systematic analysis for the Global Burden of Disease Study 2017. Lancet, 392(10159), 1923-1994. doi:10.1016/S0140-6736(18)32225-6 
6. Fu, R. J., Wang, W. X., Tao, H. J., Wang, M., Chen, Y. Y., Gao, H., . . Tang, Y. P. (2021). Quantitative evaluation of Danqi tablet by ultra-performance liquid chromatography coupled with triple quadrupole mass spectrometry integrated with bioassay. J Sep Sci, 44(7), 1552-1563. doi:10.1002/jssc.202000932

7. He, C., Li, J., Xu, N., Wang, R., Li, Z., Yang, L., \& Wang, Z. (2015). Pharmacokinetics, bioavailability, and metabolism of Notoginsenoside Fc in rats by liquid chromatography/electrospray ionization tandem mass spectrometry. J Pharm Biomed Anal, 109, 150-157. doi:10.1016/j.jpba.2015.02.038

8. Hollopeter, G., Jantzen, H. M., Vincent, D., Li, G., England, L., Ramakrishnan, V., ... Conley, P. B. (2001). Identification of the platelet ADP receptor targeted by antithrombotic drugs. Nature, 409(6817), 202207. doi:10.1038/35051599

9. Irfan, M., Jeong, D., Kwon, H. W., Shin, J. H., Park, S. J., Kwak, D., . . Rhee, M. H. (2018). GinsenosideRp3 inhibits platelet activation and thrombus formation by regulating MAPK and cyclic nucleotide signaling. Vascul Pharmacol, 109, 45-55. doi:10.1016/j.vph.2018.06.002

10. Jagadeeswaran, P., Cooley, B. C., Gross, P. L., \& Mackman, N. (2016). Animal Models of Thrombosis From Zebrafish to Nonhuman Primates: Use in the Elucidation of New Pathologic Pathways and the Development of Antithrombotic Drugs. Circ Res, 118(9), 1363-1379. doi:10.1161/CIRCRESAHA.115.306823

11. Kunapuli, S. P., Ding, Z., Dorsam, R. T., Kim, S., Murugappan, S., \& Quinton, T. M. (2003). ADP receptors-targets for developing antithrombotic agents. Curr Pharm Des, 9(28), 2303-2316. doi:10.2174/1381612033453947

12. Li, R. L., Wang, J. X., Chai, L. J., Guo, H., Wang, H., Chen, L., . . Wang, S. X. (2020). Xueshuantong for injection alleviates dtreptozotocin-Induced diabetic retinopathy in rats. Chin J Integr Med, 26(11), 825-832. doi:10.1007/s11655-020-3088-5

13. Li, Z., Delaney, M. K., O'Brien, K. A., \& Du, X. (2010). Signaling during platelet adhesion and activation. Arterioscler Thromb Vasc Biol, 30(12), 2341-2349. doi:10.1161/ATVBAHA.110.207522

14. Liu, Y., Liu, T., Ding, K., Liu, Z., Li, Y., He, T., .. . Song, X. (2018). Phospholipase Cgamma2 Signaling Cascade Contribute to the Antiplatelet Effect of Notoginsenoside Fc. Front Pharmacol, 9, 1293. doi:10.3389/fphar.2018.01293

15. Ma, L. J., Liu, F., Zhong, Z. F., \& Wan, J. B. (2017). Comparative study on chemical components and anti-inflammatory effects of Panax notoginseng flower extracted by water and methanol. J Sep Sci, 40(24), 4730-4739. doi:10.1002/jssc.201700641

16. Ma, L. J., Ma, N., Cao, J. L., \& Wan, J. B. (2022). Characterizing the influence of different drying methods on chemical components of Panax notoginseng leaves by heart-cutting two-dimensional liquid chromatography coupled to orbitrap high-resolution mass spectrometry. Food Chem, 369, 130965. doi:10.1016/j.foodchem.2021.130965

17. Michelson, A. D. (2010). Antiplatelet therapies for the treatment of cardiovascular disease. Nat Rev Drug Discov, 9(2), 154-169. doi:10.1038/nrd2957 
18. Morello, F., Perino, A., \& Hirsch, E. (2009). Phosphoinositide 3-kinase signalling in the vascular system. Cardiovasc Res, 82(2), 261-271. doi:10.1093/cvr/cvn325

19. Neuman, H., Forsythe, P., Uzan, A., Avni, O., \& Koren, O. (2018). Antibiotics in early life: dysbiosis and the damage done. FEMS Microbiol Rev, 42(4), 489-499. doi:10.1093/femsre/fuy018

20. Schror, K. (1995). Antiplatelet drugs. A comparative review. Drugs, 50(1), 7-28. doi:10.2165/00003495-199550010-00002

21. Sharma, G., \& Berger, J. S. (2011). Platelet activity and cardiovascular risk in apparently healthy individuals: a review of the data. J Thromb Thrombolysis, 32(2), 201-208. doi:10.1007/s11239-0110590-9

22. Song, F., Zhu, Y., Shi, Z., Tian, J., Deng, X., Ren, J., . . Yang, Y. (2014). Plant food anthocyanins inhibit platelet granule secretion in hypercholesterolaemia: Involving the signalling pathway of PI3K-Akt. Thromb Haemost, 112(5), 981-991. doi:10.1160/TH13-12-1002

23. Sun, H., Ma, L. J., Wan, J. B., \& Tong, S. (2021). Preparative separation of gypenoside XVII, ginsenoside Rd2, and notoginsenosides Fe and Fd from Panax notoginseng leaves by countercurrent chromatography and orthogonality evaluation for their separation. J Sep Sci, 44(15), 2996-3003. doi:10.1002/jssc. 202100078

24. Wang, L., Li, Z., Zhao, X., Liu, W., Liu, Y., Yang, J., . . Cheng, Y. (2013). A network study of chinese medicine xuesaitong injection to elucidate a complex mode of action with multicompound, multitarget, and multipathway. Evid Based Complement Alternat Med, 2013, 652373. doi:10.1155/2013/652373

25. Wang, Y., Qian, P., Liu, P., Wei, L., Cao, M., Zhou, L., . . Lin, Z. X. (2012). Effects of Panax notoginseng flower extract on the TGF-beta/Smad signal transduction pathway in heart remodeling of human chymase transgenic mice. Mol Med Rep, 5(6), 1443-1448. doi:10.3892/mmr.2012.856

26. Xu, Q. F., Fang, X. L., \& Chen, D. F. (2003). Pharmacokinetics and bioavailability of ginsenoside Rb1 and Rg1 from Panax notoginseng in rats. J Ethnopharmacol, 84(2-3), 187-192. doi:10.1016/s03788741(02)00317-3

27. Xu, X. R., Zhang, D., Oswald, B. E., Carrim, N., Wang, X., Hou, Y., . . Ni, H. (2016). Platelets are versatile cells: New discoveries in hemostasis, thrombosis, immune responses, tumor metastasis and beyond. Crit Rev Clin Lab Sci, 53(6), 409-430. doi:10.1080/10408363.2016.1200008

28. Ya, F., Li, Q., Wang, D., Xie, S., Song, F., Gallant, R. C., . . Yang, Y. (2018). Cyanidin-3-o-beta-Glucoside Induces Megakaryocyte Apoptosis via PI3K/Akt- and MAPKs-Mediated Inhibition of NF-kappaB Signalling. Thromb Haemost, 118(7), 1215-1229. doi:10.1055/s-0038-1656551

29. Ya, F., Xu, X. R., Shi, Y., Gallant, R. C., Song, F., Zuo, X., . . Yang, Y. (2019). Coenzyme Q10 Upregulates Platelet cAMP/PKA Pathway and Attenuates Integrin alphallbbeta3 Signaling and Thrombus Growth. Mol Nutr Food Res, 63(23), e1900662. doi:10.1002/mnfr.201900662

30. Yao, Y., Chen, Y., Adili, R., McKeown, T., Chen, P., Zhu, G., . . Yang, Y. (2017). Plant-based Food Cyanidin-3-Glucoside Modulates Human Platelet Glycoprotein VI Signaling and Inhibits Platelet Activation and Thrombus Formation. J Nutr, 147(10), 1917-1925. doi:10.3945/jn.116.245944 
31. Zengin, G., Ferrante, C., Gnapi, D. E., Sinan, K. I., Orlando, G., Recinella, L., . . Menghini, L. (2019). Comprehensive approaches on the chemical constituents and pharmacological properties of flowers and leaves of American basil (Ocimum americanum L). Food Res Int, 125, 108610.

doi:10.1016/j.foodres.2019.108610

32. Zhou, X., Li, Z. C., Chen, P. P., \& Xie, R. F. (2019). Primary Mechanism Study of Panax notoginseng Flower (Herb) on Myocardial Infarction in Rats. Cardiol Res Pract, 2019, 8723076. doi:10.1155/2019/8723076

33. Zuo, X., Li, Q., Ya, F., Ma, L. J., Tian, Z., Zhao, M., .. Y Yang, Y. (2021). Ginsenosides Rb2 and Rd2 isolated from Panax notoginseng flowers attenuate platelet function through P2Y12-mediated cAMP/PKA and PI3K/Akt/Erk1/2 signaling. Food Funct, 12(13), 5793-5805. doi:10.1039/d1 fo00531f

\section{Figures}
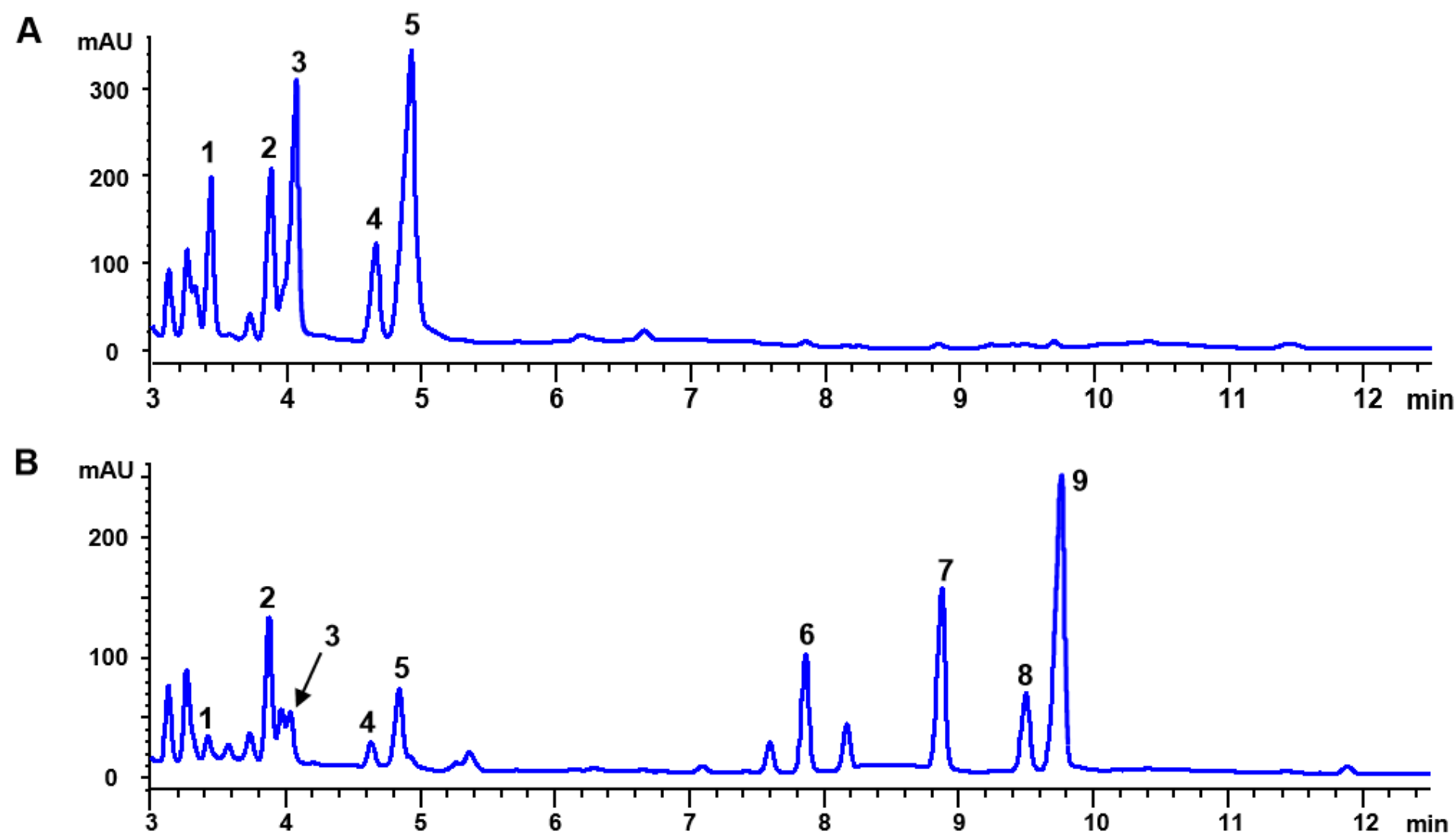

\section{Figure 1}

The UPLC-UV profiles of (A) PNFM, and (B) PNFW. 1, Ginsenoside Rb1; 2, Ginsenoside Fc; $\mathbf{3}$, GinsenosideRc; 4, Ginsenoside Rb2; 5, Ginsenoside Rb3; 6, GY- XVII; 7, notoginsenoside Fe; 8 , Ginsenoside Rd2; 9, notoginsenosideFd. 
A

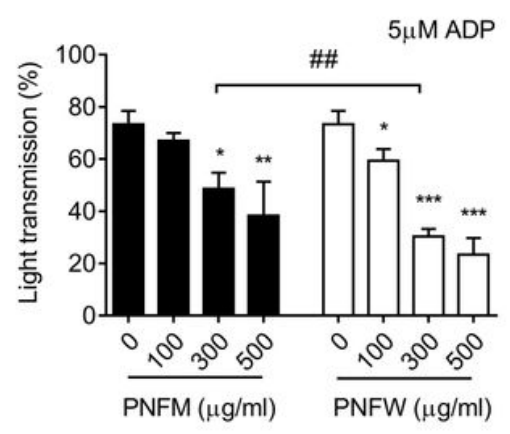

B

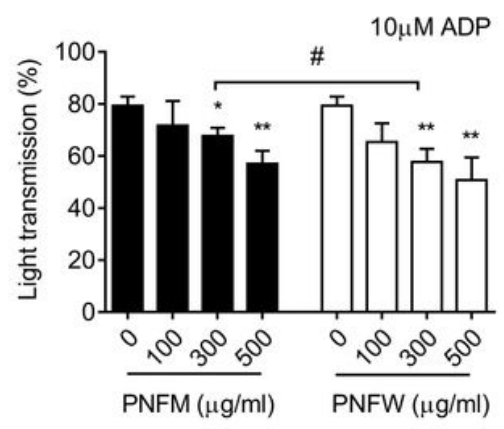

C

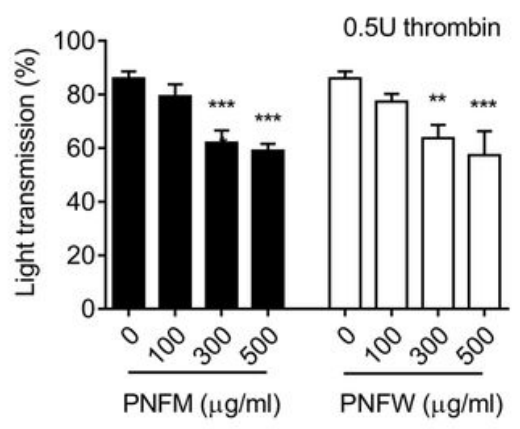

Figure 2

Effects of PNFM and PNFW on human platelet aggregation induced by ADP and thrombin. Human platelet-rich plasma (PRP) or gel-filtered platelets were pre-incubated with control buffer or the indicated doses $(100,300$, and $500 \mu \mathrm{g} / \mathrm{mL})$ of $P$. notoginseng flowers extracted by methanol (PNFM) and water (PNFW) for $20 \mathrm{~min}$ at $37^{\circ} \mathrm{C}$. A total of $250 \mathrm{~mL}$ of each sample was added to an aggregation cuvette and incubated for $1 \mathrm{~min}$. Aggregation was induced by $5 \mu \mathrm{M}$ and $10 \mu \mathrm{M}$ of adenosine $5^{\prime}$-diphosphate (ADP) or $0.5 \mathrm{U}$ thrombin and data were expressed as a percentage of maximum aggregation. Values are mean \pm $\mathrm{SD}, \mathrm{n}=4 .{ }^{\star} p<0.05,{ }^{\star \star} p<0.01$ and ${ }^{\star \star \star} p<0.001$, as compared to control buffer.

A

$200 \mu \mathrm{M}$ ADP

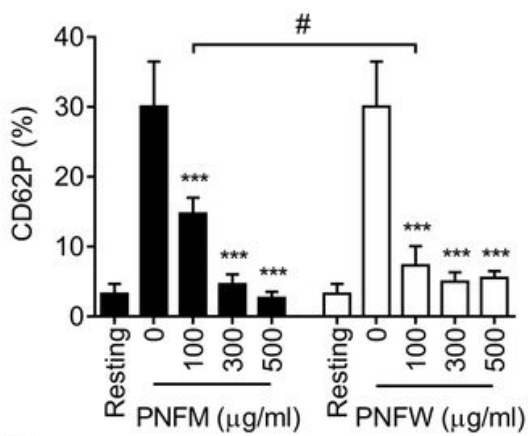

D

$0.5 \mathrm{U}$ thrombin

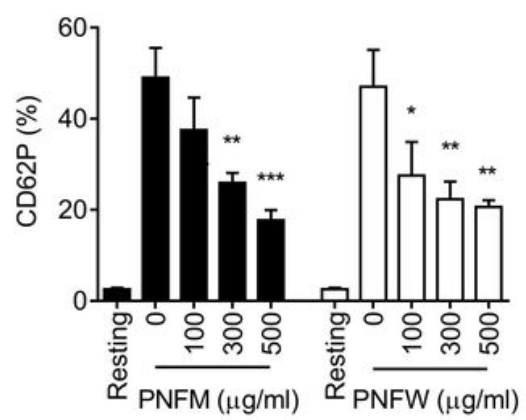

B

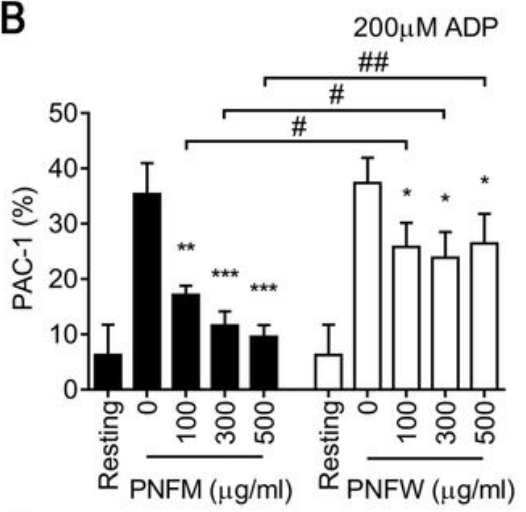

E

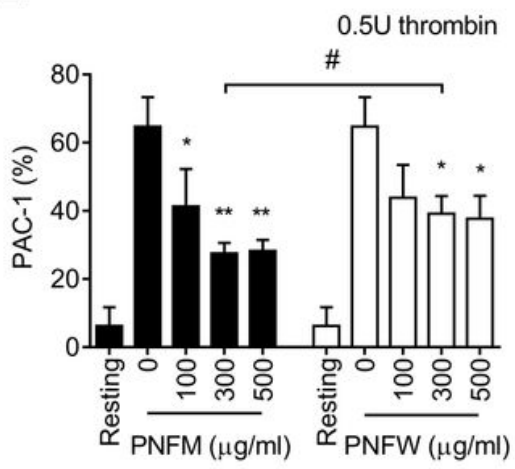

C

$200 \mu \mathrm{M}$ ADP

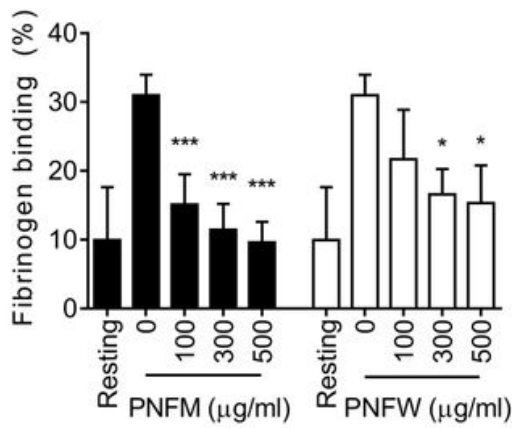

$\mathrm{F}$

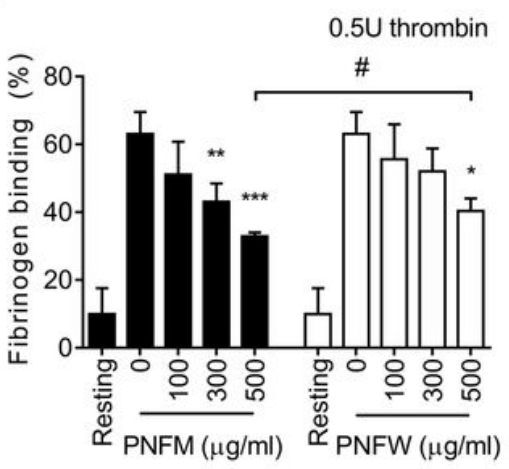


Figure 3

Effects of PNFM and PNFW on human platelet activation. Human platelet-rich plasma (PRP) or gelfiltered platelets were pre-incubated with control buffer or indicated doses $(100,300$, and $500 \mu \mathrm{g} / \mathrm{mL})$ of PNFM or PNFW for 20 min at $37^{\circ} \mathrm{C}$. Effects of PNFM and PNFW on CD62P expression, allb $\beta 3$ activation and fibrinogen binding were measured by flow cytometry analysis. Values are mean $\pm S D, n=4$. ${ }^{*} p<0.05$, ${ }^{* \star} p<0.01$ and ${ }^{* \star *} p<0.001$, as compared to control buffer.

A

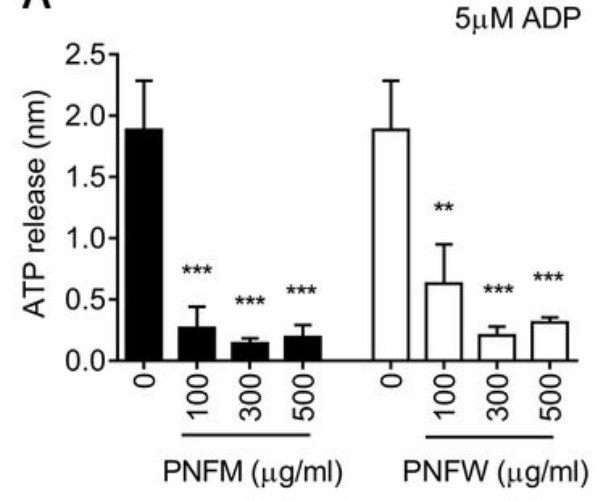

D

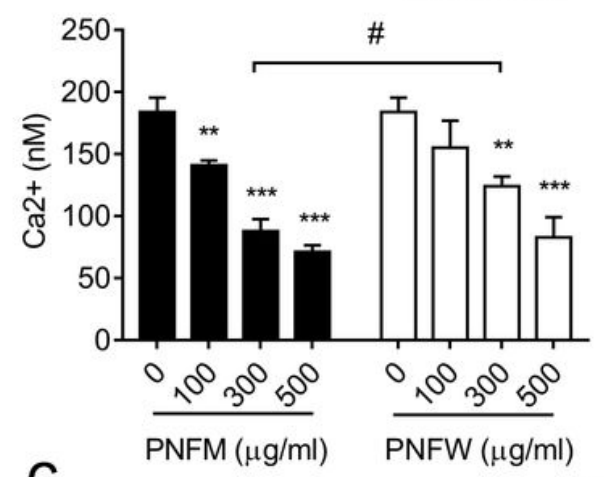

B

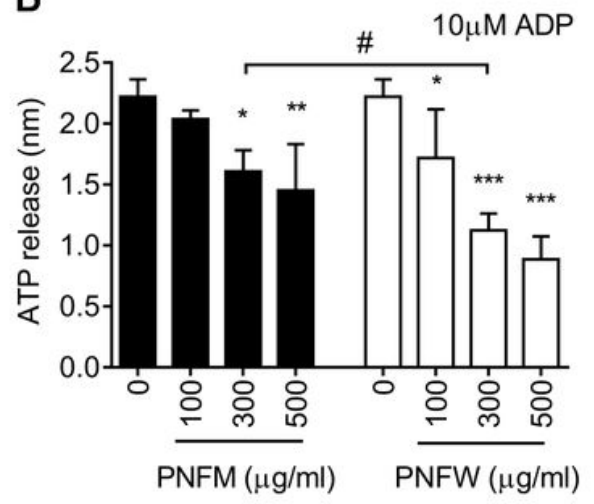

$\mathrm{E}$

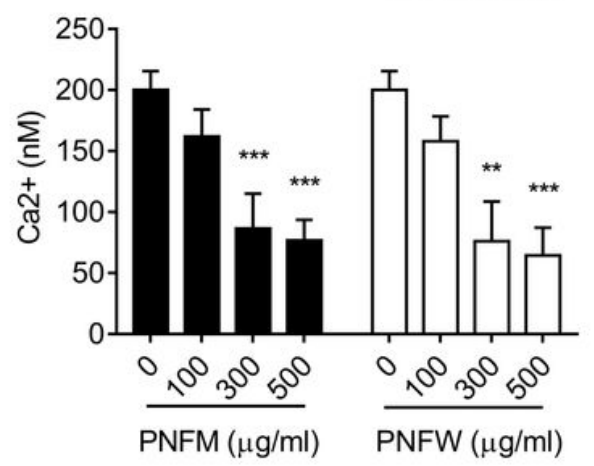

C

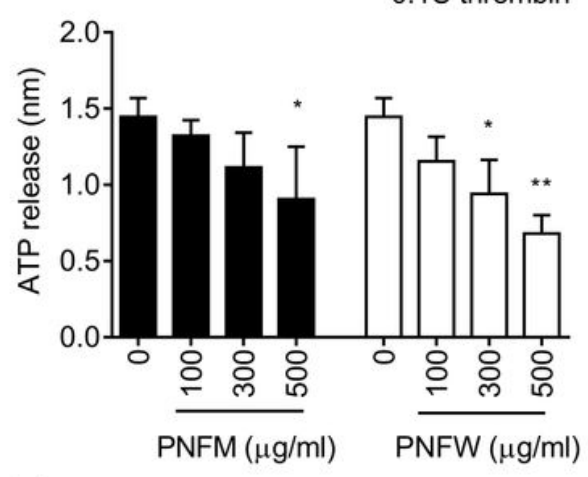

F

$5 \mu \mathrm{M}$ ADP

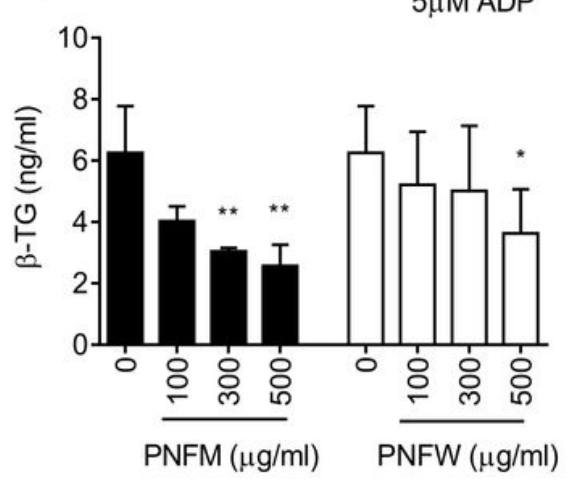

PNFM $(\mu \mathrm{g} / \mathrm{ml})$
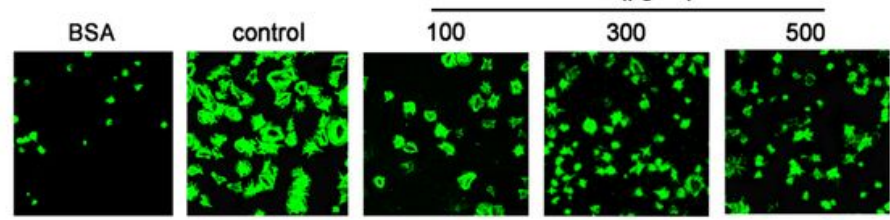

PNFW ( $\mu \mathrm{g} / \mathrm{ml})$

BSA

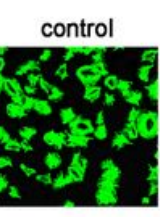

100
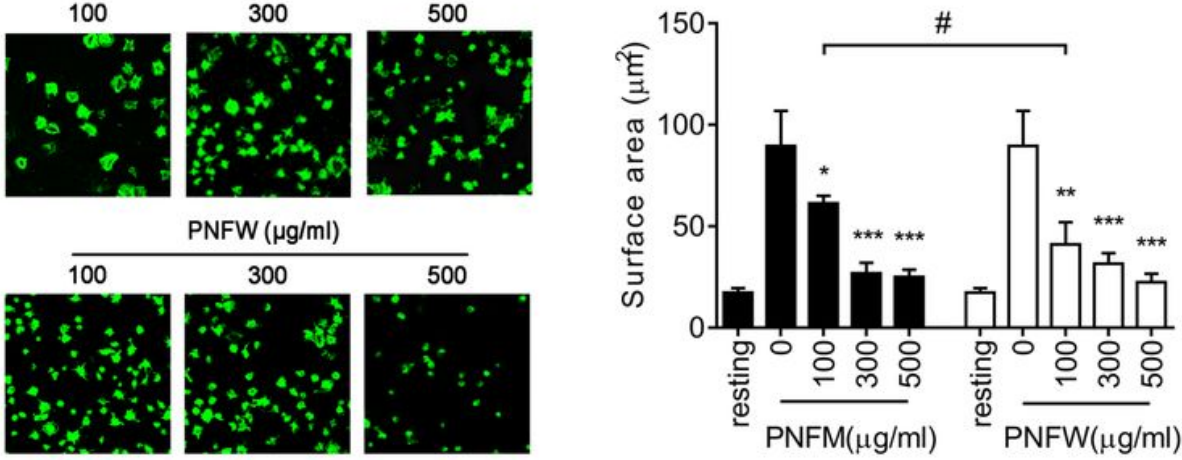

Figure 4 
Effects of PNFM and PNFW on human platelet ATP release, $\beta$-TG expression, Ca2+ mobilization and platelet spreading on immobilized fibrinogen. Human PRP or gel-filtered platelets were pre-incubated with control buffer or the indicated doses $(100,300$, and $500 \mu \mathrm{g} / \mathrm{mL})$ of PNFM and PNFW for $40 \mathrm{~min}$ at $37^{\circ} \mathrm{C}$. (A-C) Adenosine triphosphate (ATP) release induced by $5 \mu \mathrm{M}$ or $10 \mu \mathrm{M}$ ADP and $0.1 \mathrm{U}$ thrombin; (D-E) Effects of PNFM and PNFW on human platelet $\mathrm{Ca}^{2+}$ mobilization. (F) Effects of PNFM and PNFW on human platelet $\beta$-thrombomodulin ( $\beta$-TG) expression induced by $5 \mu \mathrm{M}$ ADP. (G) Effects of PNFM and PNFW on human platelet spreading. The platelets were allowed to adhere and spread on fibrinogencoated wells at $37^{\circ} \mathrm{C}$ for $1 \mathrm{~h}$, and adherent platelets were observed with an inverted fluorescence microscope. Values are mean $\pm \mathrm{SD}, \mathrm{n}=4 .{ }^{*} p<0.05$, ${ }^{\star \star} p<0.01$ and ${ }^{\star \star \star} p<0.001$, as compared to control buffer. 
A

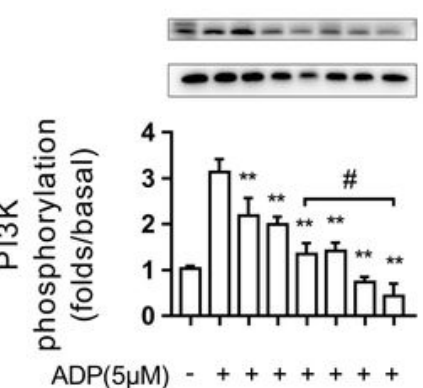

$\operatorname{PNFM}(\mu \mathrm{g} / \mathrm{ml}) \ldots+++\ldots$

$\operatorname{PNFW}(\mu \mathrm{g} / \mathrm{ml}) \ldots \ldots+++$
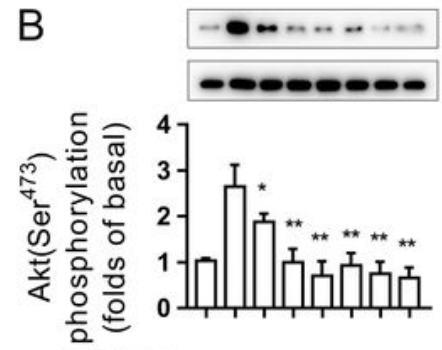

$\operatorname{ADP}(5 \mu \mathrm{M})-++++++$

$\operatorname{PNFM}(\mu \mathrm{g} / \mathrm{ml}) \cdots+++\cdots$

$\operatorname{PNFW}(\mu \mathrm{g} / \mathrm{ml}) \ldots+.++$

C

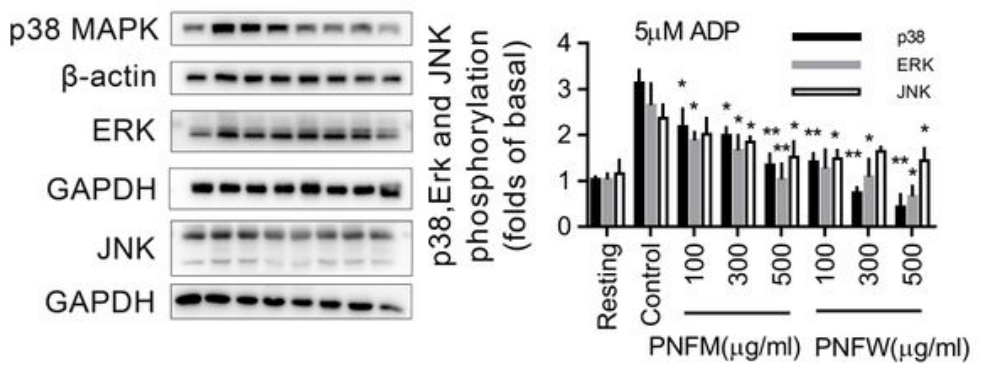

D
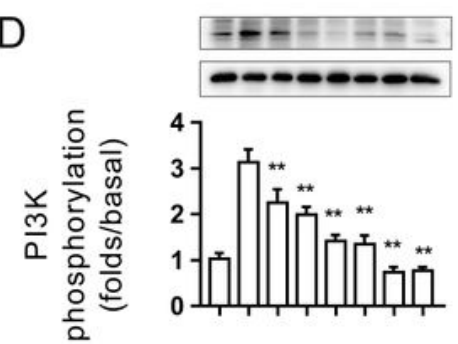

Thrombin(0.5U) -+++++++

$\operatorname{PNFM}(\mu \mathrm{g} / \mathrm{ml}) \ldots+++\cdots$

$\operatorname{PNFW}(\mu \mathrm{g} / \mathrm{ml}) \ldots \ldots+++$

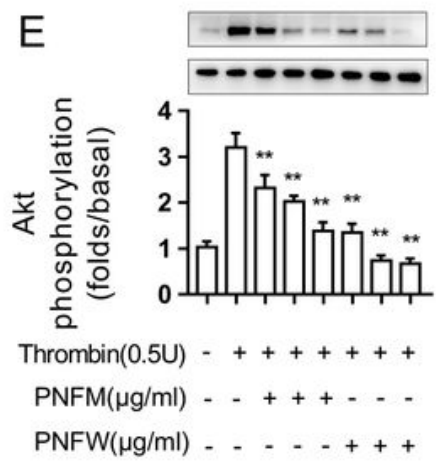

$\mathrm{F}$
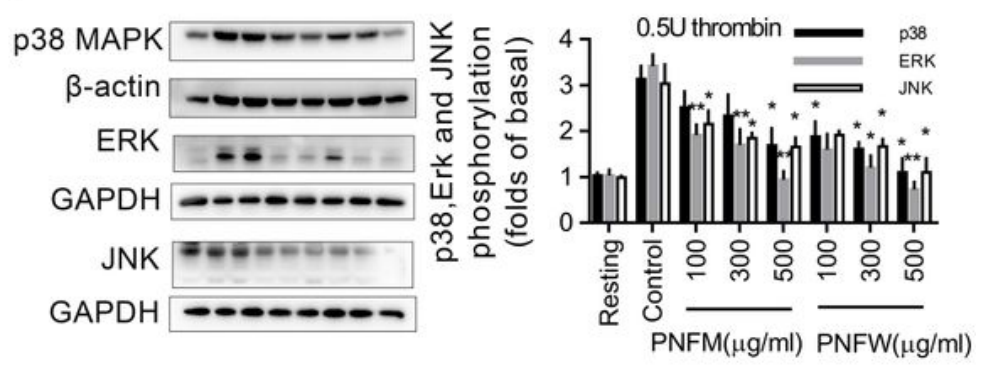

\section{Figure 5}

Effects of PNFM and PNFW on platelet PI3k Akt and P38 MAPK phosphorylation. PRP or gel-filtered platelets were initially treated with indicated doses of PNFM, PNFW or vehicle, followed by activation with $5 \mu \mathrm{M}$ ADP or $0.5 \mathrm{U}$ thrombin. Platelet were collected and the cell lysates were analyzed for PI3k Akt and P38 MAPK, Erk and JNK activation. Data are presented as mean \pm SD. ${ }^{\star \star} p<0.01$ and ${ }^{\star \star \star} p<0.001$, as compared to the ADP or thrombin control group. 


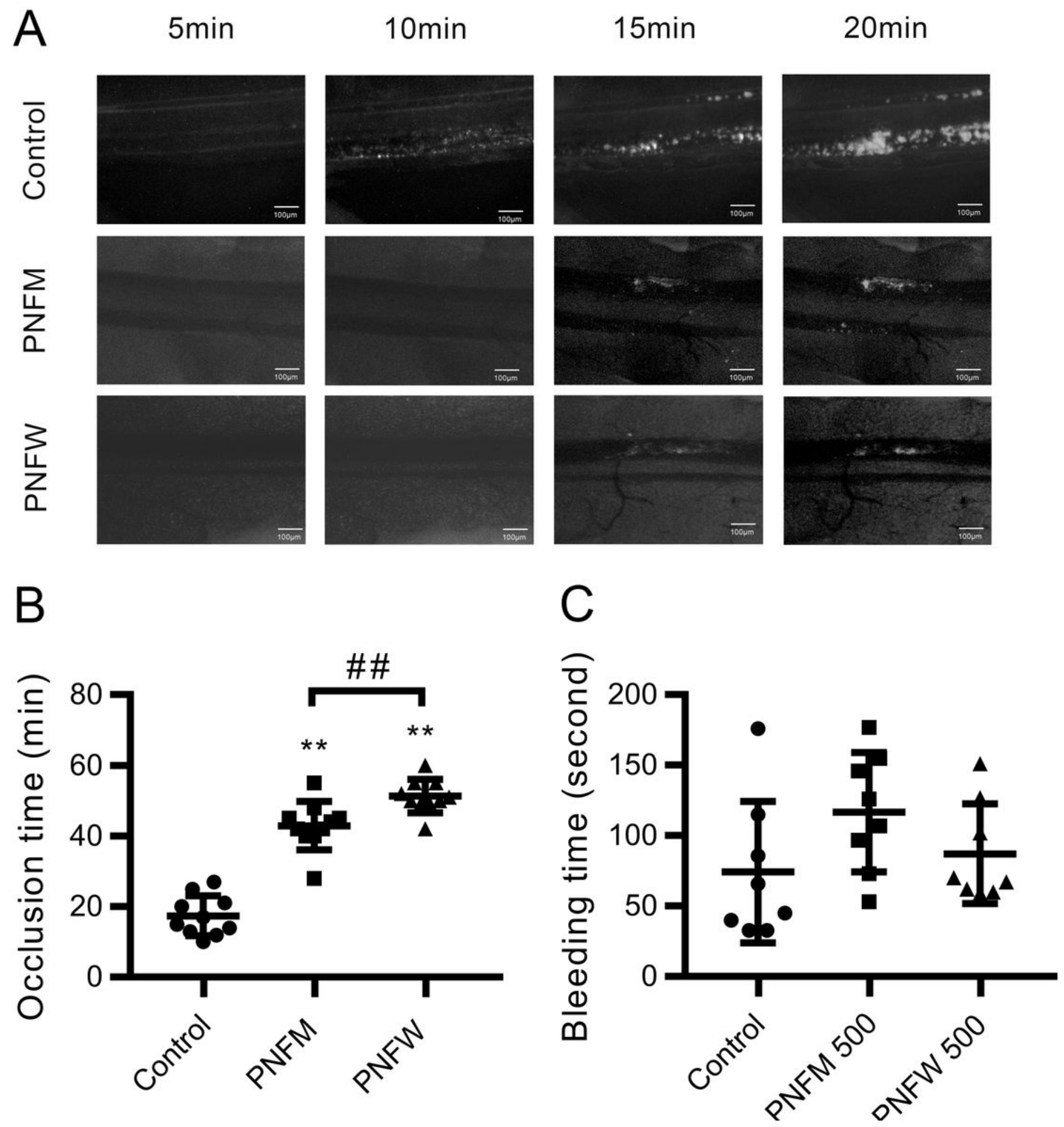

Figure 6

Effects of PNFM, PNFW on thrombosis formation and bleeding time. (A-B) Thrombus formation was initiated by topical application of $\mathrm{FeCl}_{3}$ on mesenteric arterioles in $\mathrm{C} 57 \mathrm{BL} / 6$ male mice, which were injected with fluorescent-labeled platelets and different extracts or control buffer. Thrombus formation was compared between groups based on the time to complete vessel occlusion. Values are mean $\pm S D, n$ 
$=10 .{ }^{* \star \star} p<0.001$, as compared to control. (C) Tail-vein bleeding times were examined in C57BL/6 mice. Either control buffer or PNFM/PNFW were administered via the tail vein $20 \mathrm{~min}$ before the bleeding time was determined. Values are mean $\pm S D, n=8$.

\section{Supplementary Files}

This is a list of supplementary files associated with this preprint. Click to download.

- Supplementarydata.docx 